\title{
Chrysin attenuates allergic airway inflammation by modulating the transcription factors T-bet and GATA-3 in mice
}

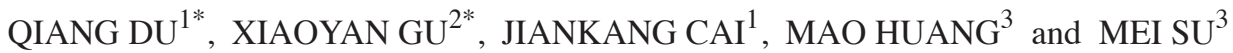 \\ ${ }^{1}$ Department of Respiratory Medicine, The Second Affiliated Hospital of Nanjing Medical University, Nanjing, \\ Jiangsu 210011; ${ }^{2}$ Department of Respiratory Medicine, The 454th Hospital of Chinese PLA, Nanjing, Jiangsu 210002; \\ ${ }^{3}$ Department of Respiratory Medicine, The First Affiliated Hospital of Nanjing Medical University, \\ Nanjing, Jiangsu 210029, P.R. China
}

Received January 17, 2012; Accepted April 20, 2012

DOI: $10.3892 / \mathrm{mmr} .2012 .893$

\begin{abstract}
Chrysin, a flavonoid obtained from various natural sources, has been reported to possess anti-inflammatory, antitumor, antioxidant and anti-allergic activities. However, its anti-inflammatory and immunoregulatory activities in asthma animal models are poorly understood. In the present study, we examined the effects of chrysin on airway inflammation and the possible mechanisms through which it acts in a murine model of allergic asthma. BALB/c mice sensitized and challenged to ovalbumin (OVA) were administered intragastrically with chrysin at a dose of $50 \mathrm{mg} / \mathrm{kg}$ daily. Chrysin significantly suppressed OVA-induced airway hyperresponsiveness (AHR) to acetylcholine chloride (Ach). Chrysin administration significantly inhibited the total inflammatory cell and eosinophil counts in bronchoalveolar lavage fluid (BALF) and total immunoglobulin $\mathrm{E}$ (IgE) levels in serum. Histological examination of lung tissue demonstrated that chrysin significantly attenuated allergeninduced lung eosinophilic inflammation and mucus-producing goblet cells in the airway. In addition, chrysin triggered a switch of the immune response to allergens towards a T-helper type 1 (Th1) profile by modulating the transcription factors T-bet and GATA-3 in allergic mice. These data suggest that chrysin exhibits anti-inflammatory and immunoregulatory properties and provides new insights into the immunopharmacological role of chrysin in terms of its effects in a murine model of asthma.
\end{abstract}

\section{Introduction}

Allergic asthma is a chronic pulmonary disease associated with airway hyperresponsiveness (AHR) and chronic inflam-

Correspondence to: $\mathrm{Dr} \mathrm{Mei} \mathrm{Su}$, Department of Respiratory Medicine, The First Affiliated Hospital of Nanjing Medical University, 300 Guangzhou Road, Nanjing, Jiangsu 210029, P.R. China E-mail: sumei2033@163.com

*Contributed equally

Key words: chrysin, asthma, airway inflammation, T-bet, GATA-3 mation of the airways (1). A previous study showed that allergic airway inflammation is associated with increased T-helper type 2 (Th2) cytokine production and decreased T-helper type 1 (Th1) cytokine production (2). The transcription factors GATA-3 and T-bet are important in Th cytokine production; GATA-3 increased Th2 cytokine production in asthma and the loss of T-bet may be associated with asthma (3-4).

Current treatment guidelines for asthma emphasize anti-inflammatory therapy, and glucocorticoids remain the first-line agents. Even though glucocorticoids are effective in preventing allergic inflammation, few asthmatic patients are able to obtain normal bronchial reactivity, even after years of steroid therapy. Moreover, patients with chronic asthma treated with inhaled glucocorticoids, particularly in the long-term, may develop serious side effects (5-6). Thus there is a need for a new or alternative approach to manage this disease.

Chrysin (5,7-dihydroxyflavone), a natural flavonoid obtained from many plants, honey and propolis, possesses extensive pharmacological activities such as anti-inflammatory, anti-allergy and immunoregulatory effects via the suppression of inducible nitric oxide synthase and nuclear factor- $\kappa \mathrm{B}$ (7-10). Since chrysin affects the immune system, it may be able to be used to treat asthma. The aim of the present study was to investigate the role of chrysin on the allergic airway inflammation and Th1/Th2 cytokine production modulated by T-bet and GATA-3 in a murine model of allergic asthma.

\section{Materials and methods}

Experimental animal. A total of 48 six-week-old female $\mathrm{BALB} / \mathrm{c}$ mice, weighing 18-22 $\mathrm{g}$ each, were purchased from the Shanghai Laboratory Animal Center (Shanghai, China). All experimental animals used in this study were maintained under a protocol approved by the Institutional Animal Care and Use Committee of the Nanjing Medical University and the experimental protocol was approved by the institutional animal ethics committee.

Allergen sensitization/challenge protocol. Mice were randomly divided equally into 4 groups: Control, ovalbumin (OVA), chrysin and budesonide. Each group contained 12 mice, 6 for pulmonary resistance and 6 for bronchoalveolar lavage 
(BAL). Mice in the last three groups were sensitized on days 0 and 14 by intraperitoneal injection of $100 \mu \mathrm{g}$ OVA (Grade V; Sigma, St Louis, MO, USA) emulsified in $1 \mathrm{mg}$ aluminum hydroxide (Pierce Chemical Co., Rockford, IL, USA) with a total volume of $0.2 \mathrm{ml}$. Mice were challenged via the airway with $1 \%$ OVA daily on days 22, 23 and 24. The OVA challenge was performed for $30 \mathrm{~min}$ by placing the mice in a Plexiglas box $(29 \times 22 \times 18 \mathrm{~cm})$ and aerosolizing OVA using an ultrasonic nebulizer (NE-U11B, Omuron, Tokyo, Japan). Mice in the chrysin group were also administered intragastrically with chrysin (Sigma) at a dose of $50 \mathrm{mg} / \mathrm{kg}$ (11-13) daily from days 15 to 24 on consecutive days. Mice in the budesonide group were exposed to aerosolized budesonide $(2 \mathrm{mg}, 1 \mathrm{mg} / 2 \mathrm{ml}$, Astra Zeneca) for $30 \mathrm{~min}$ per day from days 22 to $24,1 \mathrm{~h}$ prior to challenge. Mice in the control group received the same schedule for sensitization and challenge with an equivalent amount of $0.9 \%$ sterile saline instead of OVA.

Measurement of airway responsiveness to acetylcholine chloride (Ach). For the measurement of airway responsiveness to Ach, the mice were anaesthetized by intraperitoneal injection of pentobarbital sodium $(70 \mathrm{mg} / \mathrm{kg}) 24 \mathrm{~h}$ after the last challenge. A plastic tube of $2 \mathrm{~mm}$ in internal diameter was inserted into the trachea via tracheotomy and a 27 gauge needle was inserted into the caudalis vein for drug administration. The mice were then placed in a whole body plethysmography chamber and ventilated mechanically at a rate of 90 breaths $/ \mathrm{min}$ with a tidal volume of $6 \mathrm{ml} / \mathrm{kg}$ with an AniRes animal lung function analysis system (Synol High-Tech, Beijing, China). Following the establishment of stable airway pressure recording, ACh was administered intravenously with a microinfusion pump at a rate of $36 \mathrm{ml} / \mathrm{h}$ in progressively increasing doses $(10,30,90$ and $270 \mu \mathrm{g} / \mathrm{kg})$. Following the administration of each dose, data were continuously collected from $5 \mathrm{sec}$ to $1 \mathrm{~min}$ and maximum values of lung resistance $(\mathrm{RL})$ were taken to express the changes in the airway function of the mice (14-15).

Analysis of bronchoalveolar lavage fluid (BALF) samples and serum. Blood samples were collected $24 \mathrm{~h}$ after the last aerosol challenge by retroorbital puncture using heparinized capillary tubes. Blood samples were centrifuged $\left(10 \mathrm{~min}, 4^{\circ} \mathrm{C}, 1000 \mathrm{x} \mathrm{g}\right)$, and plasma was stored at $-70^{\circ} \mathrm{C}$ until further use. The lungs were washed three times with $0.5 \mathrm{ml}$ saline to collect BALF. The BALF was centrifuged $\left(10 \mathrm{~min}, 4^{\circ} \mathrm{C}, 1000 \mathrm{x} \mathrm{g}\right)$, and the total number of inflammatory cells in BALF was counted with a hemocytometer. Smears of BAL cells were stained with Wright's staining for the differential cell count. The cells in the BALF were counted by two independent investigators in a single-blind study, which analyzed at least 200 cells each from four different random locations using a microscope. The levels of IL- 4 and IFN- $\gamma$ (Jingmei Biotech Company, Shanghai, China) in BALF and total serum IgE (Bionewtrans Pharmaceutical Biotechnology Co. Ltd., Franklin, MA, USA) were determined by ELISA according to the manufacturer's instructions. The limits of detection were $7 \mathrm{pg} / \mathrm{ml}$ for IL-4, $7 \mathrm{pg} / \mathrm{ml}$ for IFN- $\gamma$ and $1 \mathrm{ng} / \mathrm{ml}$ for total $\mathrm{IgE}$.

Lung histology. For the histological evaluation of lung tissue, the right lungs obtained from sacrificed mice were immersed in
$10 \%$ formaldehyde overnight, and then embedded in paraffin. A series of micro sections $(5 \mu \mathrm{m})$ were cut on a microtome and stained with hematoxylin and eosin (H\&E) to assess the inflammatory cell infiltration, and Periodic acid Schiff (PAS) to quantify airway global cells. Scoring for mucus production in the PAS-stained sections was as follows (16): 0, no goblet cells; $1,<25 \%$ of the epithelium; $2,25-50 \%$ of the epithelium; $3,50-75 \%$ of the epithelium; $4,>75 \%$ of the epithelium. At least 5 bronchioles in each slide that were randomly distributed throughout the left lung were analyzed. Scoring was undertaken by two individuals blinded to the experimental protocol.

Measurement of T-bet and GATA-3 protein by western blot analysis. The left lung tissues from each group were added to lysis buffer and homogenized. After absolute schizolysis and centrifugation at $12000 \mathrm{x}$ g for $15 \mathrm{~min}$ at $4^{\circ} \mathrm{C}$, the resulting supernatants were collected as the total protein samples. The protein concentrations were determined using the method of bicinchoninic acid. In total, $50 \mu \mathrm{g}$ of total proteins isolated from the whole lung were separated by $10 \%$ sodium dodecyl sulfate-polyacrylamide gel electrophoresis and blotted onto a nitrocellulose membrane for $2 \mathrm{~h}$. The membrane was then incubated in blocking solution (5\% dry milk in Tris-buffered saline) for $1 \mathrm{~h}$ at room temperature and subsequently exposed to $0.8 \mu \mathrm{g} / \mathrm{ml}$ of the monoclonal antibodies anti-GATA-3, anti-T-bet or anti- $\beta$-actin (Cell Signaling Technology, Beverly, MA, USA) overnight at $4{ }^{\circ} \mathrm{C}$ with gentle agitation. The following day, the membrane was incubated with peroxidase-conjugated goat antimouse $\operatorname{IgG}(1: 1000)$ for $2 \mathrm{~h}$ at room temperature. Protein was detected by the ECL Plus (Amersham Biosciences, Piscataway, NJ, USA). Densitometric analysis was performed by ImageJ densitometry software $(\mathrm{NIH})$ after scanning of the films. Target protein levels were normalized to $\beta$-actin protein levels.

Statistical analysis. Data were shown as the means \pm standard error of the mean (SEM). Statistical analysis was performed using one-way analysis of variance (ANOVA) and the post hoc least significant difference (LSD) test with SPSS software (SPSS, Chicago, IL, USA). $\mathrm{P}<0.05$ was considered to indicate a statistically significant difference.

\section{Results}

Chrysin suppresses airway hyperreactivity in OVA sensitized mice. The airway resistance in anaesthetized mice was measured using invasive whole body plethysmography (Fig. 1). There were no significant differences in baseline airway resistance among the four groups. The airway resistance generated by the administration of Ach at doses from 30 to $270 \mu \mathrm{g} / \mathrm{kg}$ increased significantly in the OVA-sensitized/challenged mice compared with the control mice. The OVA group had significantly greater airway resistance than the control group. Treatment with chrysin and budesonide resulted in a sharp decrease in airway resistance compared with the OVA group.

Chrysin attenuates allergic airway inflammation in mice. Aside from macrophages, few inflammatory cells were detected in control BALB/C mice. Sensitization and challenge with OVA resulted in a marked increase of total leukocyte and 


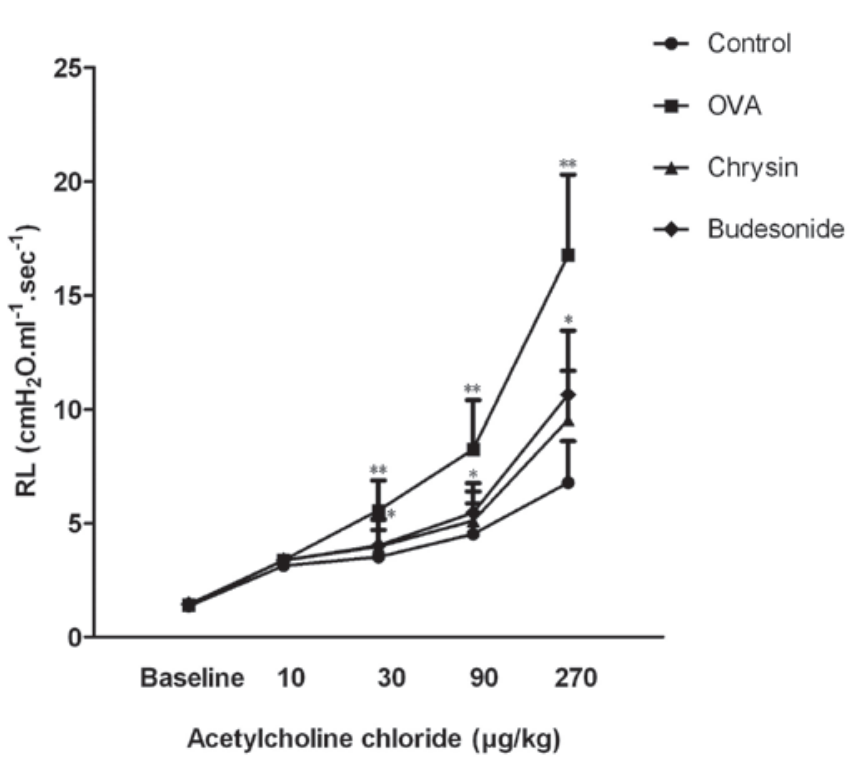

Figure 1. Treatment with chrysin decreased the development of AHR in a murine model of asthma. Data are the means \pm SEM ( $n=6$ per group). ${ }^{* *} \mathrm{P}<0.01$ compared with the control group; ${ }^{\mathrm{P}} \mathrm{P}<0.05$ compared with the OVA group. AHR, airway hyperresponsiveness; OVA, ovalbumin.

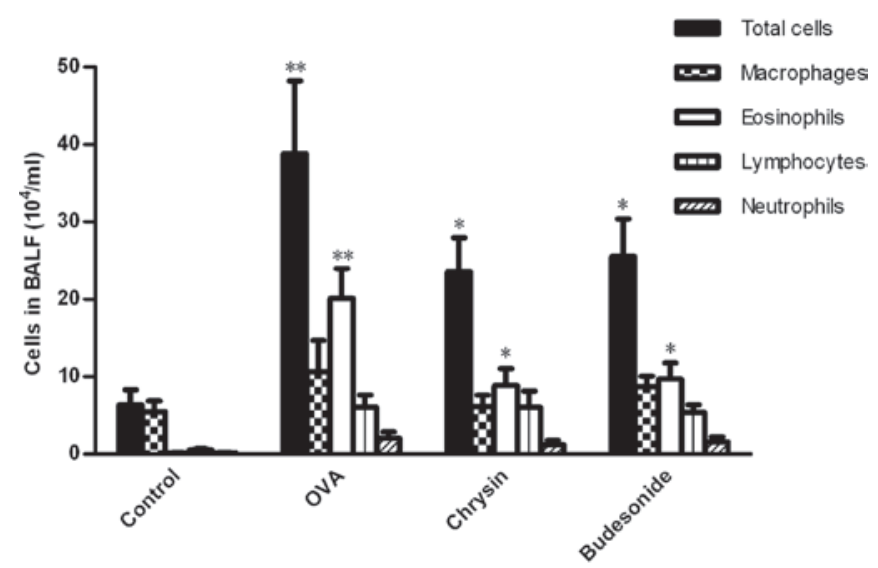

Figure 2. Treatment with chrysin inhibited the inflammatory cell accumulation in broncho-alveolar lavage fluid (BALF) in a murine model of asthma. Data are the means \pm SEM ( $=6$ per group). ${ }^{* *} \mathrm{P}<0.01$ compared with the control group; ${ }^{*} \mathrm{P}<0.05$ compared with the OVA group. OVA, ovalbumin.

eosinophil counts in BALF. The number of total inflammatory cells and eosinophils were significantly decreased in BALF following treatment with chrysin (Fig. 2). The anti-inflammatory effect of chrysin was demonstrated following histological examination of the H\&E- and PAS-stained sections. A significant change of inflammatory cells in the airway and around the blood vessels was observed in OVA-sensitized/challenged mice, but not in the saline-treated control mice. The majority of the infiltrated inflammatory cells were eosinophils. Chrysin significantly attenuated eosinophil-rich inflammatory leukocyte infiltration compared with OVA-challenged mice (Fig. 3).

Chrysin inhibits airway goblet cell hyperplasia and mucus production in a murine model of asthma. Marked goblet
Table I. Cytokine levels in BALF and serum total IgE were measured by ELISA.

\begin{tabular}{lccc}
\hline Group & IL-4 (pg/ml) & IFN- $\gamma(\mathrm{pg} / \mathrm{ml})$ & IgE $(\mathrm{ng} / \mathrm{ml})$ \\
\hline Control & $19.02 \pm 6.65$ & $28.72 \pm 5.28$ & $53.8 \pm 14.21$ \\
OVA & $119.15 \pm 32.43^{\mathrm{a}}$ & $14.62 \pm 3.25^{\mathrm{a}}$ & $226.0 \pm 26.83^{\mathrm{a}}$ \\
Chrysin & $55.23 \pm 9.38^{\mathrm{b}}$ & $24.85 \pm 4.32^{\mathrm{b}}$ & $98.0 \pm 22.61^{\mathrm{b}}$ \\
Budesonide & $53.70 \pm 10.25^{\mathrm{b}}$ & $23.35 \pm 5.14^{\mathrm{b}}$ & $105.7 \pm 19.15^{\mathrm{b}}$
\end{tabular}

Treatment with chrysin enhances the level of IFN- $\gamma$ and reduces the levels of the Th2-type cytokine IL-4 in BALF and total serum IgE in a murine model of asthma. Data are the means \pm SEM ( $n=6$ per group). ${ }^{\mathrm{a}} \mathrm{P}<0.05$ compared with the control group, ${ }^{\mathrm{b}} \mathrm{P}<0.05$ compared with the OVA group. BALF, bronchoalveolar lavage fluid; IgE, immunoglobulin E; OVA, ovalbumin.

cell hyperplasia and mucus hypersecretion in the bronchi were observed in the OVA-challenged mice, but not in the control mice. Chrysin significantly attenuated OVA-induced overproduction of mucus and goblet cell hyperplasia in mice (Fig. 4a-d). PAS staining scores were calculated for each experimental group, as described in Materials and methods. Mucus secretion was markedly increased in mice in the OVA group with enhanced staining scores (Fig. 4e). There was a significant reduction in the proportion of goblet cells in the airway of mice administered chrysin.

Chrysin alters the levels of Th1/Th2 cytokines in BALF and total $\mathrm{IgE}$ in serum. BALF was collected $24 \mathrm{~h}$ after the last OVA challenge, and the concentration of IFN- $\gamma$ and IL-4 were assessed using ELISA. The level of the Th2 cytokine IL-4 in BALF was significantly increased, but the concentration of IFN- $\gamma$ was decreased in the OVA group compared with the control group $(\mathrm{P}<0.05$, Table I). Total serum IgE levels were significantly elevated in the OVA group compared with the control group. Treatment with chrysin reduced total serum IgE levels compared with the OVA group $(\mathrm{P}<0.05$; Table I).

Chrysin alters the ratio of T-bet and GATA-3 in the lung. The relative amount of target protein was quantified as the density of the target protein versus $\beta$-actin. Following normalization of the data according to the expression of $\beta$-actin protein, the relative density of T-bet and GATA-3 are presented as fold numbers (Fig. 5A). The ratio of T-bet and GATA-3 was significantly decreased in the OVA group compared with the control group $(\mathrm{P}<0.01$; Fig. 5B). Treatment with chrysin markedly increased the ratio of T-bet and GATA-3 compared with OVA-sensitized/challenged mice ( $\mathrm{P}<0.05$; Fig. 5B).

\section{Discussion}

In the current study, the effects of chrysin on allergy-induced airway inflammation in a murine model of allergic asthma were evaluated. The findings have shown that chrysin attenuated OVA-induced airway inflammation and mucus secretion in the lung tissues of allergic mice. Chrysin administration enhanced IFN- $\gamma$ and decreased IL-4 levels in BALF and total 


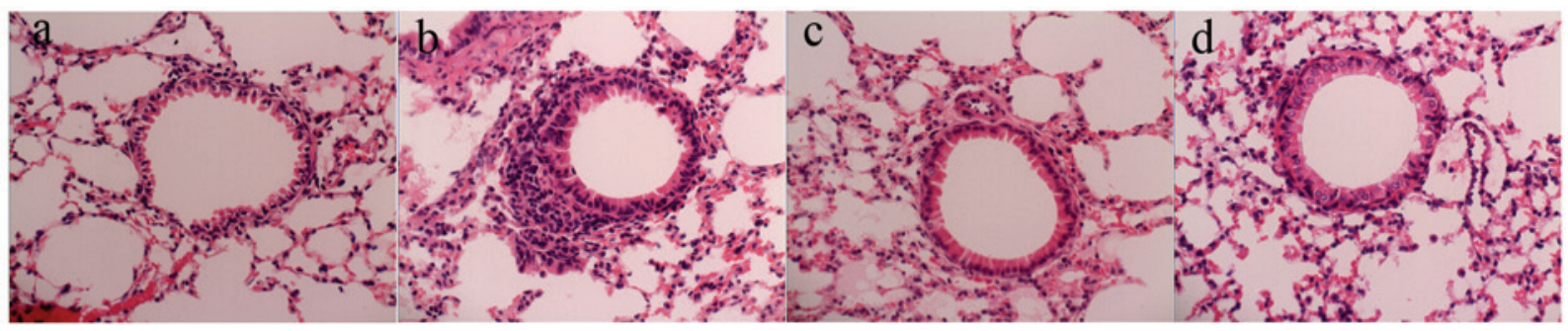

Figure 3. Treatment with chrysin attenuates airway inflammation in a murine model of asthma. Hematoxylin and eosin staining (original magnification, x100) of: (a) Control; (b) OVA; (c) chrysin; and (d) budesonide groups. OVA, ovalbumin.
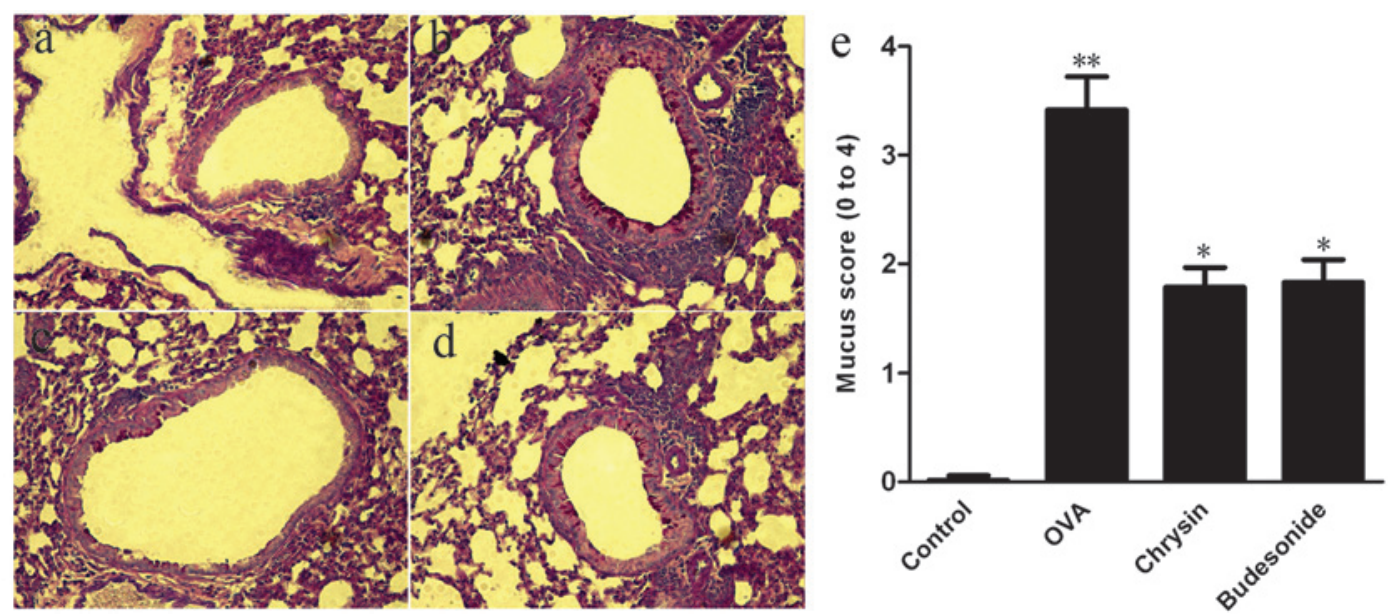

Figure 4. Treatment with paeonol inhibits antigen-induced mucus production in a murine model of asthma. Periodic acid Schiff (PAS) staining (original magnification, x200) of: (a) Control; (b) OVA; (c) chrysin; and (d) budesonide groups. (e) Quantitative analyses of mucus production in lung sections were performed as described in Materials and methods. Data are the means \pm SEM ( $n=6$ per group). ${ }^{* *} \mathrm{P}<0.01$ compared with the control group; ${ }^{*} \mathrm{P}<0.05$ compared with the OVA group. OVA, ovalbumin.

A

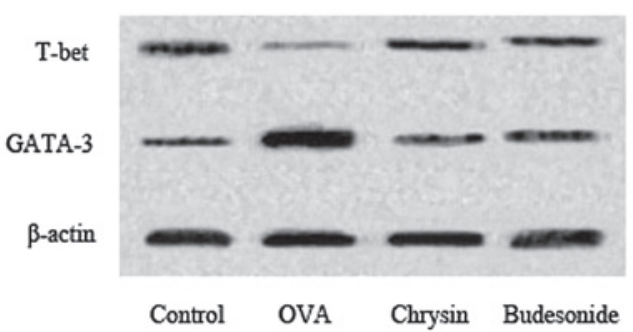

B

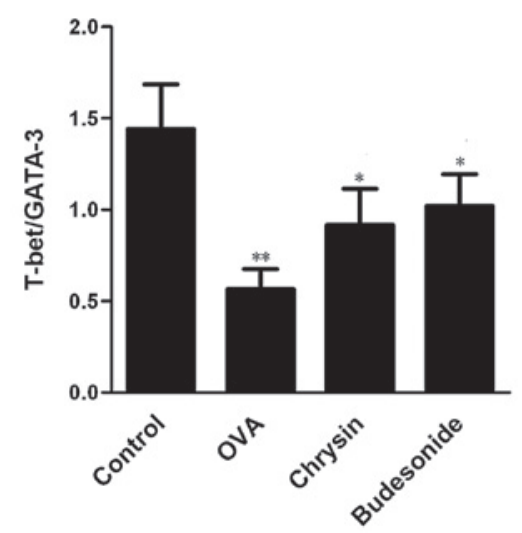

Figure 5. Treatment with chrysin increased the ratio of T-bet and GATA-3 in the lung in a murine model of asthma. Treatment with chrysin (A) increased the relative density of T-bet and decreased the relative density of GATA-3, and (B) increased the ratio of T-bet and GATA-3 compared with the OVA group. Data are the means \pm SEM ( $n=6$ per group). ${ }^{* *} \mathrm{P}<0.01$ compared with the control group; ${ }^{*} \mathrm{P}<0.05$ compared with the OVA group. OVA, ovalbumin.
IgE in serum. In addition, treatment with chrysin regulates the level of T-bet and GATA-3 levels in lungs. Furthermore, we showed that chrysin markedly suppressed AHR in a murine model of asthma.

A previous study has demonstrated the regulation effect of chrysin on the immune system in mice, as evidenced by the decreased levels of total IgE and Th2 cytokines (17). In asthma, allergen-induced Th2 cells in the lungs lead to eosinophilic airway inflammation, increased secretion of mucus and recurring bronchospasm (18). In this study, we observed that treatment with chrysin markedly reduced not only the eosinophil count, but also the Th2 cytokine IL-4 level in BALF. Lung histology also validated the fact that chrysin suppressed the eosinophilic airway inflammation and mucus-producing goblet cells in the airway. These results suggest that chrysin is an anti-inflammatory or anti-allergic herbal extract that appears to be beneficial for the treatment of allergic asthma.

It is widely accepted that $\operatorname{Th} 1 / \mathrm{Th} 2$ imbalance plays a significant role in asthma. $\mathrm{CD} 4^{+}$Th cells can be divided into Th1 and Th2 groups defined by functional differences and cytokine profiles. Induction of the transcription factors T-bet and GATA-3 play crucial roles in Th cell differentiation into Th2 and Th1 cells (19-20). T-bet, a member of the T-box family of transcription factors, was a master determinant of Th1 lineage, and T-bet-deficient mice exhibited a profound 
lack of Th1 immune responses (21). GATA-3 is a transcription factor that is specifically expressed in Th2 cells and plays a critical role in the differentiation of Th2 cells from uncommitted $\mathrm{CD} 4^{+}$lymphocytes. It has been reported that IFN- $\gamma$ and IL-4 levels in the lymphocytes of asthmatic patients were correlated with the ratio of T-bet/GATA-3, which may be used as an objective indicator of immune imbalance in patients with allergic asthma (21). Therefore, resetting the T-bet/GATA-3 ratio imbalance may have an important therapeutic role for asthma (22-24). In this study, the ratio of T-bet to GATA-3 was markedly decreased accompanied by an increase of the Th2 cytokine IL-4 and inhibition of synthesis of the Th1 cytokine IFN- $\gamma$ in the asthma group compared with the control group, and partly reverted in the chrysin treatment group.

The pathophysiology of AHR is complex and a number of factors contribute to its development. Chronic airway inflammation is an important factor that results in AHR. Th2 cytokines, particularly IL-4, which regulate inflammatory cell recruitment to the lung, are critical in allergic inflammation and the development of AHR (25-26). Administration of the Th1 cytokine IFN- $\gamma$ effectively attenuates established allergyinduced airway inflammation and AHR in mice (27). In addition, eosinophils are crucial in the development of AHR, since AHR is absent in mice whose eosinophils are completely ablated (28). Our findings demonstrated, for the first time, that treatment with chrysin significantly inhibited AHR in a model of allergic asthma. The inhibition of AHR may be attributed to restoring the Th1/Th2 imbalance and the reduction of eosinophilic airway inflammation in allergic mice.

In conclusion, our data have shown that treatment with chrysin reduces airway inflammation and AHR due to the alteration of Th1/Th2 polarization via the suppression of GATA-3 and the increase of T-bet expression in OVA-induced allergic mice. Therefore, the use of chrysin is a promising approach towards a novel asthma therapy.

\section{Acknowledgements}

This work was supported by grants from the Key Project of Science and Technology Development Foundation of Nanjing Medical University (09NMUZ23).

\section{References}

1. Bousquet J, Jeffery PK, Busse WW, Johnson M and Vignola AM: Asthma: from bronchoconstriction to airways inflammation and remodelling. Am J Respir Crit Care Med 161: 1720-1745, 2000.

2. Holgate ST: The epidemic of allergy and asthma. Nature 402 Suppl: B2-B4, 1999.

3. Finotto S, Neurath MF, Glickman JN, et al: Development of spontaneous airway changes consistent with human asthma in mice lacking T-bet. Science 295: 336-342, 2002.

4. Zheng W and Flavell RA: The transcription factor GATA-3 is necessary and sufficient for Th2 cytokine gene expression in CD4 ${ }^{+} \mathrm{T}$ cells. Cell 89: 587-596, 1997.

5. Foster JM, Sanderman R, van der Molen T, Mueller T and van Sonderen E: Personality influences the reporting of side effects of inhaled corticosteroids in asthma patients. J Asthma 45: 664-669, 2008

6. Irwin RS and Richardson ND: Side effects with inhaled corticosteroids: the physician's perception. Chest 130 (Suppl 1): S41-S53, 2006.

7. Ha SK, Moon E and Kim SY: Chrysin suppresses LPS-stimulated proinflammatory responses by blocking NF- $\mathrm{KB}$ and JNK activations in microglia cells. Neurosci Lett 485: 143-147, 2010.
8. Hougee S, Sanders A, Faber J, Graus YM, van den Berg WB, Garssen J, Smit HF and Hoijer MA: Decreased pro-inflammatory cytokine production by LPS-stimulated PBMC upon in vitro incubation with the flavonoids apigenin, luteolin or chrysin, due to selective elimination of monocytes/macrophages. Biochem Pharmacol 69: 241-248, 2005.

9. Wadibhasme PG, Ghaisas MM and Thakurdesai PA: Anti-asthmatic potential of chrysin on ovalbumin-induced bronchoalveolar hyperresponsiveness in rats. Pharm Biol 49: 508-515, 2011.

10. Bae Y,Lee S and Kim SH: Chrysin suppresses mast cell-mediated allergic inflammation: involvement of calcium, caspase- 1 and nuclear factor-кB. Toxicol Appl Pharmacol 254: 56-64, 2011.

11. Zhai K, Hu L, Chen J, Fu CY and Chen Q: Chrysin induces hyperalgesia via the GABAA receptor in mice. Planta Med 74: 1229-1234, 2008

12. Pushpavalli G, Kalaiarasi P, Veeramani C and Pugalendi KV: Effect of chrysin on hepatoprotective and antioxidant status in D-galactosamine-induced hepatitis in rats. Eur J Pharmacol 631: $36-41,2010$.

13. Zhang S, Wang X, Sagawa K and Morris ME: Flavonoids chrysin and benzoflavone, potent breast cancer resistance protein inhibitors, have no significant effect on topotecan pharmacokinetics in rats or mdrla/lb (-/-) mice. Drug Metab Dispos 33: 341-348, 2005.

14. Du Q, Chen Z, Zhou LF, Zhang Q, Huang M and Yin KS: Inhibitory effects of astragaloside IV on ovalbumin-induced chronic experimental asthma. Can J Physiol Pharmacol 86: 449-457, 2008

15. Du Q, Zhou LF, Chen Z, Gu XY, Huang M and Yin KS: Imiquimod, a toll-like receptor 7 ligand, inhibits airway remodelling in a murine model of chronic asthma. Clin Exp Pharmacol Physiol 36: 43-48, 2009.

16. Lee MY, Ahn KS, Kwon OK, et al: Anti-inflammatory and anti-allergic effects of kefir in a mouse asthma model. Immunobiology 212: 647-654, 2007.

17. Yano S, Umeda D, Yamashita T, et al: Dietary flavones suppresses IgE and Th2 cytokines in OVA-immunized BALB/c mice. Eur J Nutr 46: 257-263, 2007.

18. Cohn L, Homer RJ, Marinov A, Rankin J and Bottomly K: Induction of airway mucus production by $\mathrm{T}$ helper $2(\mathrm{Th} 2)$ cells: a critical role for interleukin 4 in cell recruitment but not mucus production. J Exp Med 186: 1737-1747, 1997.

19. Barnes PJ: Role of GATA-3 in allergic diseases. Curr Mol Med 8: 330-334, 2008

20. Finotto $\mathrm{S}$ and Glimcher L: T cell directives for transcriptional regulation in asthma. Springer Semin Immunopathol 25: 281-294, 2004.

21. Yong J, Chen GQ, Huang B and Wu S: Correlation between the ratio of T-bet/GATA-3 and the levels of IL- 4 and IFN- $\gamma$ in patients with allergic asthma. Mol Med Report 4: 663-666, 2011.

22. Kiwamoto T, Ishii Y, Morishima Y, et al: Transcription factors T-bet and GATA-3 regulate development of airway remodeling. Am J Respir Crit Care Med 174: 142-151, 2006.

23. Bian T, Yin KS, Jin SX, et al: Treatment of allergic airway inflammation and hyperresponsiveness by imiquimod modulating transcription factors T-bet and GATA-3. Chin Med J (Engl) 119: 640-648, 2006

24. Li RR, Pang LL, Du Q, Shi Y, Dai WJ and Yin KS: Apigenin inhibits allergen-induced airway inflammation and switches immune response in a murine model of asthma. Immunopharmacol Immunotoxicol 32: 364-370, 2010.

25. Kips JC, Tournoy KG and Pauwels RA: New anti-asthma therapies: suppression of the effect of interleukin (IL)-4 and IL-5. Eur Respir J 17: 499-506, 2001

26. Cohn L, Tepper JS and Bottomly K: IL-4-independent induction of airway hyperresponsiveness by Th2, but not Th1 cells. J Immunol 161: 3813-3816, 1998

27. Behera AK, Kumar M, Lockey RF and Mohapatra SS: Adenovirus-mediated interfereon gamma gene therapy for allergic asthma: involvement of interleukin 12 and STAT4 signaling. Hum Gene Ther 13: 1697-1709, 2002.

28. Lee JJ, Dimina D, Macias MP, et al: Defining a link with asthma in mice congenitally deficient in eosinophils. Science 305: 1773-1776, 2004. 